Elsevier required licence: (c) <2019>. This manuscript version is made available under the CC-BY-NC-ND 4.0 license http://creativecommons.org/licenses/by-nc-nd/4.0/

The definitive publisher version is available online at $h$ ttps://doi.org/10.1016/j.jes.2018.03.003 


\title{
Dynamic membrane bioreactor performance enhancement by powdered activated carbon addition: Evaluation of sludge morphological, aggregative and microbial properties
}

\author{
Zhenzhen $\mathrm{Yu}^{1}$, Yisong $\mathrm{Hu}^{1,3}$, Mawuli Dzakpasu ${ }^{3}$, Xiaochang C. Wang ${ }^{1,2,3}{ }_{\star}$, Huu Hao Ngo ${ }^{3,4}$ \\ 1. Key Lab of Northwest Water Resource, Environment and Ecology, MOE, Xi'an University of Architecture and Technology, Xi'an 710055, China \\ 2. Key Lab of Environmental Engineering, Shaanxi Province, Xi'an 710055, China \\ 3. International Science \& Technology Cooperation Center for Urban Alternative Water Resources Development, Xi'an 710055, China \\ 4. Centre for Technology in Water and Wastewater, School of Civil and Environmental Engineering, University of Technology Sydney, Sydney, \\ NSW 2007, Australia \\ * Corresponding author. E-mail: xcwang@xauat.edu.cn (Xiaochang C. Wang)
}

\begin{abstract}
The effects of powdered activated carbon (PAC) addition on sludge morphological, aggregative and microbial properties in a dynamic membrane bioreactor (DMBR) were investigated to explore the enhancement mechanism of pollutants removal and filtration performance. Sludge properties were analyzed through various analytical measurements. The results showed that the improved sludge aggregation ability and the evolution of microbial communities affected sludge morphology in PAC-DMBR, as evidenced by the formation of large, regularly shaped and strengthened sludge flocs. The modifications of sludge characteristics promoted the formation process and filtration flux of the dynamic membrane (DM) layer. Additionally, PAC addition did not exert very significant influence on the propagation of eukaryotes (protists and metazoans) and microbial metabolic activity. High-throughput pyrosequencing results indicated that adding PAC improved the bacterial diversity in activated sludge, as PAC addition brought about additional microenvironment in the form of biological PAC (BPAC), which promoted the enrichment of Acinetobacter (13.9\%), Comamonas (2.9\%), Flavobacterium (0.31\%) and Pseudomonas (0.62\%), all contributing to sludge flocs formation and several (such as Acinetobacter) capable of biodegrading relatively complex organics. Therefore, PAC addition could favorably modify sludge properties from various aspects and thus enhance the DMBR performance.
\end{abstract}

\section{Keywords}

Dynamic membrane bioreactor; Powdered activated carbon; Sludge property; Filtration performance; Wastewater treatment; Microbial community

Introduction

Conventional membrane bioreactor (MBR) encounters the main challenges of high cost of membrane module and membrane fouling (Tian et al., 2011; Yu et al., 2017; Zhao et al., 2000). The dynamic membrane bioreactor (DMBR) with the notable advantages of low capital cost, less energy demand, higher flux and easy cleaning has emerged as an alternative to the conventional MBR (Hu et al., 2016; Quek et al., 2017). The so-called dynamic membrane (DM) layer in the DMBR process is defined as a cake 
layer and/or gel layer that forms on the large pore support material (10-200 $\mu \mathrm{m})$ by filtering suspended solid particles such as sludge flocs.

Generally, the operation of the DMBR includes three stages: DM layer formation, stable filtration and cleaning for DM regeneration (Hu et al., 2016). The proper control of the three filtration stages is crucial for achieving excellent DMBR performance, which is still needed to be investigated due to the following reasons. Firstly, the DM layer formation time should be further shortened, which is beneficial for minimizing the production of effluent with poor quality in the initial DM filtration stage because of the insufficient rejection by support material itself. Moreover, the DM layer is susceptible to the drastic hydraulic turbulence, which can influence the stability of the DM structure and should be avoided during the bioreactor operation (Kiso et al., 2000). Thus, the development of a stable and highly permeable DM layer is crucial to achieve the long stable DMBR operation.

Some additives (such as PAC) are able to enhance DMBR performance by modifying the sludge properties such as dewaterability, settleability, flocculability, extracellular polymeric substances (EPSs), particle size distribution (PSD) and viscosity (Chu et al., 2013; Hu et al., 2017; Satyawali and Balakrishnan, 2009). It was reported that PAC addition in the DMBR shortened the DM formation time, increased the stable effluent flux and enhanced the pollutant removal at low PAC dosages $(\leq 3 \mathrm{~g} / \mathrm{L})$. These effects were mainly attributed to the formation of the biological PAC (BPAC) particles, by which simultaneous adsorption and biodegradation were achieved (Hu et al., 2017).

In addation to the aforementioned sludge properties, the effects of PAC on other sludge properties, such as morphology (particle size and shape) and aggregation ability, remain largely not well understood in the PAC-DMBR hybrid process. As reported, in a lab-scale MBR system, Meng et al. (2006) claimed that the irregular shape of sludge flocs caused a severe cake fouling, resulting in a negative effect on the membrane filtration process. Moreover, Su et al. (2013) studied the effect of sludge aggregation ability on the membrane fouling in the MBR and suggested that the sludge with poor aggregation ability aggravated cake formation on the membrane surface, reflecting the high membrane fouling potential. The aggregation ability of sludge flocs could be described by the extended DerjaguinLandau-Verwey-Overbeek (XDLVO) theory (Hou et al., 2015; Yuan et al., 2017), which stated the interaction of the two solid surface as the sum of Lifshitz-van der Waals (LW), Lewis acidbase $(\mathrm{AB})$ and electrostatic double layer (EL) interactions (Hong et al., 2016, 2017). It is believed that the investigation of the sludge morphology and aggregation ability will provide new insight into the effects of PAC addition on the PAC-DMBR operation.

Additionally, the surface and/or pores of PAC can provide a suitable environment for the microbial communities that can degrade the adsorbed organic substances for growth, which consequently, enhances the pollutants removal performance and affects the structures of microbial communities in the activated sludge (Lin et al., 2001; Skouteris et al., 2015). Furthermore, various eukaryotes (such as protists and meta-zoans) can be used as indicator organisms for the activated sludge properties, effluent quality and bioreactor performance in conventional activated sludge and MBR systems (Achmadulina et al., 2017; Derlon et al., 2013; Jenkins et al., 2004). Moreover, in
MBRs, the predation and movement behaviors of eukaryotes in the cake layer produce a more heterogeneous, open and porous structure, which increases the permeability of the cake layer ( $\mathrm{Wu}$ et al., 2017). However, the combined investigations of eukaryotes using microscopic observations and bacterial communities using modern molecular technology (such as high-throughput pyrosequencing) in PAC-DMBR system are still limited.

The objective of this study was to analyze the sludge morphological, aggregative, and microbial properties in the PAC-DMBR system with the aim to elucidate the mechanism of process performance enhancement. Analytical methods including PSD analysis, microscopic observations, fractal theory and image analysis, XDLVO theory, Biolog assay and highthroughput pyrosequencing were used to measure the sludge properties.

\section{Materials and methods}

\subsection{Experimental setup and operation}

Two identical lab-scale DMBRs located at a local wastewater treatment plant (WWTP) in Xi'an, China were operated in parallel (Appendix A Fig. S1). Each DMBR had a rectangular effective working volume of $15 \mathrm{~L}$. Four cylindrical air diffusers were placed at the bottom of the four corners to continuously create aeration and suspend the activated sludge under the actions of an air pump (at an air flow of $48 \mathrm{~L} / \mathrm{min}$ ), which produced micro-bubbles of several millimeters in diameter. A flat-sheet DM module made of polyvinyl chloride plates and support layers was vertically immersed in each reactor with details shown in Table 1. Real domestic wastewater collected from the regulating tank of the local WWTP was fed into the two DMBRs by submersible pumps. The concentrations of chemical oxygen demand (COD), ammonia $\left(\mathrm{NH}_{3}-\mathrm{N}\right)$, total phosphorus (TP), color, $\mathrm{UV}_{254}$, and turbidity in the domestic wastewater varied in the range of 130-263.8 mg/L, 20.9-29.5 mg/L, 2.9-3.7 mg/L, 102.4-155.0 c.u., $0.10-0.24 \mathrm{~cm}^{-1}$, and 13.8-37.2 NTU, respectively. The effluent was withdrawn continuously by maintaining a $10-\mathrm{cm}$ water level difference between the bioreactor and the effluent port. Under the constant pressure operation mode, a gradual decrease in the membrane flux was observed with the operation time. When the flux declined to a pre-determined value (e.g., 10\% of the initial flux) physical cleaning (such as air backwashing with a flow rate of $72 \mathrm{~L} / \mathrm{min}$ for $2 \mathrm{~min}$ ) was implemented for DM regeneration and permeability recovery (Hu et al., 2016).

Commercial PAC with the mean particle size of $29.4 \mu \mathrm{m}$ was added at $1 \mathrm{~g} / \mathrm{L}$ to one DMBR and named PAC-DMBR. The other, named C-DMBR, was operated without PAC addition to serve as a

\begin{tabular}{ll} 
Table $1-$ Properties of dynamic membrane module. \\
\hline \multicolumn{1}{c}{ Item } & \multicolumn{1}{c}{ Parameter } \\
\hline Inner support material & Stainless steel mesh \\
Pore size of inner layer $(\mathrm{mm})$ & 10 \\
Outer support material & Nylon mesh \\
Pore size of outer layer $(\mu \mathrm{m})$ & 75 \\
Single-sided effective filtration area $\left(\mathrm{m}^{2}\right)$ & 0.02 \\
Total effective filtration area $\left(\mathrm{m}^{2}\right)$ & 0.04 \\
\hline
\end{tabular}


control. Before seeding of the DMBRs, the inoculation sludge obtained from the aerobic tank of the local WWTP was acclimated with real domestic wastewater for about two weeks. Subsequently, identical MLSS of the acclimated sludge was put into the two DMBRs. Meanwhile, the commercial PAC, after cleaning with deionized water, was added into the PAC-DMBR. PAC and sludge flocs were well mixed by aerating the mixture for one day, then both DMBRs entered a short startup period (two weeks), followed by a stable operation period (one month). There was no PAC supplement except for the initial addition. Minimized PAC loss was achieved, as most of PAC quickly interacted with sludge flocs during the early stage of the startup period. Additionally, the short DM formation time alleviated the amount of PAC loss along with effluent permeation at the very beginning filtration stage.

During the stable operation period, the two DMBRs did not discharge sludge except for small amounts that were lost along with the initial effluent and were regularly sampled for sludge property measurement. The frequency of sludge sampling was once a week during the stable operation period. As a result, the sludge retention time (SRT) was approximately 100-150 days.

The average MLSS respectively were $2500 \mathrm{mg} / \mathrm{L}$ and $3500 \mathrm{mg} / \mathrm{L}$ in

the C-DMBR and PAC-DMBR. Because the membrane flux was decreased from near $510 \mathrm{~L} /\left(\mathrm{m}^{2} \cdot \mathrm{hr}\right)$ in the initial stage to $260-$ $310 \mathrm{~L} /\left(\mathrm{m}^{2} \cdot \mathrm{hr}\right)$ after the DM immediate formation, eventually ended at approximately $50 \mathrm{~L} /\left(\mathrm{m}^{2} \cdot \mathrm{hr}\right)$ for the air backwashing in the PAC-DMBR, the hydraulic retention time (HRT) of the PAC-

DMBR was in the range of $1.2-7.5 \mathrm{hr}$ after DM formation. Similarly, the HRT of the C-DMBR was calculated as $1.5-7.5 \mathrm{hr}$. The more operational parameters of the two DMBRs during the stable operation period can be found in Table 2.

\subsection{Fractal theory and image analysis}

Fractal dimension $\left(D_{P}\right)$ is often used to describe the geometric features of sludge flocs, which is determined by the perimeterarea relationship of fractal theory analysis based on image analysis. Additionally, a static light scattering method has also been proposed as a novel and reliable alternative to measure the parameter of $D_{P}$ (Zhao et al., 2016a, 2018). In this study, the former was applied because the other shape parameters of sludge floc (such as roundness (Ro), form factor (FF), and aspect ratio (AR)) also could be determined through image analysis (Meng et al., 2006). In this study, the specific operation procedures for getting these parameters were as follows. Firstly, the phase contrast images of sludge flocs were observed by a microscope (N90i,

Table 2 - Operational conditions in the two DMBRs during the stable operation period.

\begin{tabular}{lll}
\multicolumn{1}{c}{ Parameters } & C-DMBR & PAC-DMBR \\
\hline Temperature $\left({ }^{\circ} \mathrm{C}\right)$ & $18-22$ & $18-22$ \\
pH & $7.3-7.7$ & $7.3-7.7$ \\
DO (mg/L) & $2-6$ & $2-6$ \\
SRT (d) & $100-150$ & $100-150$ \\
HRT (hr) & $1.5-7.5$ & $1.2-7.5$ \\
MLSS (mg/L) & 2500 & 3500 \\
\hline
\end{tabular}

DMBR: dynamic membrane bioreactor; PAC: powdered activated carbon.

${ }^{\text {a }}$ Data was given as the average value.
Nikon Corporation, Japan) equipped with a digital camera and image analysis software (Nikon NIS-elements AR 3.2). Secondly, the acquired images of JEPG format were transformed into greylevel images using Adobe Photoshop 7.0 software (Adobe Systems Inc., San Jose, CA). In order to easily distinguish flocs from the background, two thresholds which were the maximums of the first and second derivation of the grey level histogram were set for each image (Cenens et al., 2002). Thus, a grey-level image was converted to a binary image. Next, the parameters of floc area, perimeter, length and width were determined in the binary image using Nikon NIS software. For each sludge sample, at least 50 images were analyzed to get accurate data. Finally, the shape parameters of $D_{P}$, Ro, FF, and AR were calculated by the formulae listing in Appendix A to characterize the floc morphology.

\subsection{Assessing sludge aggregation ability using XDLVO theory}

Recently, many researchers have assessed the membrane fouling and sludge aggregation ability in MBRs by XDLVO theory (Cai et al., 2017; Su et al., 2013; Zhao et al., 2016b). In the current work XDLVO theory was mainly used to assess the aggregation

ability of sludge flocs. The total interaction energy ( $U_{\text {sls }}^{\text {XDLO }}$ ) as a

function of separation distance between the sludge cells could be calculated as the sum of Lifshitz-van der Waals energy ( $\left.U_{\text {sls }}^{\mathrm{LW}}\right)$, Lewis acid-base energy $\left(\mathrm{U}_{\text {sls }}^{\mathrm{AB}}\right)$ and electrostatic double layer energy $\left(\mathrm{U}_{\mathrm{sls}}^{\mathrm{EL}}\right)$ by XDLVO theory:

$$
U_{\text {sls }}^{\mathrm{XDLVO}}=U_{\mathrm{sls}}^{\mathrm{LW}}+U_{\mathrm{sls}}^{\mathrm{AB}}+U_{\mathrm{sls}}^{\mathrm{EL}}
$$

where the subscripts "l" and "s" correspond to the liquid and solid, respectively. The calculation of the items of $U_{s l s}^{\mathrm{LW}}, U_{\mathrm{sls}}^{\mathrm{AB}}$ and $\mathrm{U}_{\mathrm{sls}}^{\mathrm{EL}}$ in the XDLVO theory is based on the surface thermodynamic approach (Hou et al., 2015). The contact angles and zeta potentials of the sludge flocs are very important for the calculation of the surface thermodynamic parameters and XDLVO theory. The measurement methods of contact angles and zeta potentials as well as detailed procedures for calculating surface thermodynamic parameters and XDLVO theory are presented in Appendix A.

\subsection{Biolog assay}

The carbon substrate utilization patterns of microbial communities in the sludge samples were obtained by the Biolog assay to determine the microbial community characteristics especially related to the community metabolic property. The commercial Biolog EcoPlate ${ }^{\mathrm{TM}}$ (BIOLOG ${ }^{\mathrm{TM}}$ Inc., Hayward, CA, USA) containing 31 different carbon sources in triplicate and 3 negative controls (without carbon source) in a 96-well plate format was used. The detailed operations mainly followed that reported in a previous study (Yang et al., 2011), but with some modifications. The incubation time was prolonged to $120 \mathrm{hr}$. Average well color development (AWCD) for all 31 carbon sources was considered as an index to evaluate the microbial average activity (Elfstrand et al., 2007), which could be calculated as follows:

$$
\text { AWCD }=\frac{\sum_{i=1}^{31}\left(R_{i t}-R_{0 t}\right)}{31}
$$


where $R_{i t}$ and $R_{0 t}$ are the absorbance values of the sole carbon source $\mathrm{i}$ and the water blank at time $\mathrm{t}$.

According to a previous study (Choi and Dobbs, 1999), the 31 carbon sources in the Biolog EcoPlate ${ }^{\mathrm{TM}}$ could be divided into six categories: polymers, carbohydrates, carboxylic acids, amine acids, amines and phenolic compounds. In order to determine the metabolic characteristic of microbial communities on the six carbon categories, the average absorbance ratio in each category was calculated and compared according to Eqs. (3) and (4) (Zhang et al., 2014):

$$
\begin{gathered}
f_{j}=\frac{1}{n_{j}} \sum_{i=1}^{n_{j}} R_{i} \\
F_{j}=\frac{f_{j}}{\sum_{i=1}^{n} f_{i}}
\end{gathered}
$$

where $f_{j}$ is the average absorbance of category $j ; F_{j}$ is the absorbance ratio of category $j ; R_{i}$ is the absorbance of carbon source $i$ which belongs to category $\mathrm{j}$ at the end-point of the reaction; and $n_{j}$ is the number of carbon source in the special category j.

\subsection{High-throughput pyrosequencing}

High-throughput pyrosequencing of 16S rRNA bacterial genes was conducted on the Illumina Miseq ${ }^{\mathrm{TM}}$ platform (Illumina Inc., San Diego, CA) to elucidate the impact of PAC addition on the bacterial community composition and abundance in the DMBR. The procedures for DNA extraction and PCR amplification were carried out before high-throughput pyrosequencing. Sludge samples from the two DMBRs at the end of the operation were analyzed. The detailed procedures are presented in Appendix A.

\subsection{Other analysis}

PSD analysis was performed using a laser granularity distribution analyzer (LS230/SVM+, Beckman Coulter Corporation, USA) with a detection range of 0.04-2000 $\mu \mathrm{m}$. Microscopic observations of eukaryotes were directly obtained through a light microscope (N90i, Nikon Corporation, Japan). The chemical parameters $\left(\mathrm{NH}_{3}{ }^{-}\right.$ $\mathrm{N}, \mathrm{TP}, \mathrm{COD}, \mathrm{UV}_{254}$ ) and some physical parameters (MLSS, MLVSS) were monitored according to Standard Methods (Chinese NEPA, 2002). Turbidity was measured with a turbidity meter (ET266020, Lovibond Corporation, Germany), color with a colorimeter (SD9011B, China), pH with a pH meter (PHS-3C, China), temperature with a thermometer (WN-G/Y-01, China), dissolved oxygen (DO) concentration with a DO meter (Model HQ30d, Hatch Corporation, USA), and the filtration flux of the DM with the volumetric method.

\section{Results and discussion}

\subsection{Performance of PAC-DMBR and C-DMBR}

As for the treatment performance, during one-month stable operation period, the influent and effluent quality of the two DMBRs was regularly analyzed, which was shown in Section 1.1 and Appendix A Table S1. It could be seen that lower concentrations of COD, $\mathrm{NH}_{3}-\mathrm{N}$, color and $\mathrm{UV}_{254}$ were detected in the PAC-
DMBR as compared with the C-DMBR. The high removals of these pollutants in the PAC-DMBR could be attributed to the significant effect of combined adsorption and biodegradation of BPAC and the certain effect of rejection properties of the deposited DM layer as a "secondary" membrane (Ersahin et al., 2012; Skouteris et al., 2015; Whang et al., 2004). However, the low removals of TP were observed in the two DMBRs, which was due to hard survive of polyphosphate-accumulating organisms under aerobic environment. Overall, it indicated that pollutant removal was enhanced by PAC addition in the PAC-DMBR.

The filtration performances of the two DMBR were also detected. Fig. 1 showed typical filtration profiles describing the cycle variations of effluent flux and turbidity with the operating time, which approximately correspond to the 4th-7th day of the stable operational period. The multiple cycles of operation (presenting the reproducibility of DM performance) can be found in Appendix A Fig. S2.

From Appendix A Fig. S2, under the constant pressure operation mode (10-cm water head), none drastic fluctuation of the effluent flux and turbidity, and other water quality items for every operation cycle was observed. The results indicated that there was no sudden partial detachment of DM layer from the surface of mesh under the given operation conditions, and thus, DM layers in the two DMBRs were considered to be stable.

Moreover, as shown in Fig. 1, for the PAC-DMBR a rapid flux decline in the initial filtration stage turned into more gradual decreasing tendency in the stable filtration stage and almost ended at flux of $50 \mathrm{~L} /\left(\mathrm{m}^{2} \cdot \mathrm{hr}\right)$. The similar trend was also obtained in the C-DMBR. However, PAC-DMBR showed higher flux than that in C-DMBR, because approximately $10 \mathrm{~L} /\left(\mathrm{m}^{2} \cdot \mathrm{hr}\right)$ higher stable operational flux was noted in the PAC-DMBR rather than in the C-DMBR. Commonly, the effluent turbidity falling below 1 NTU or an SS of zero can be considered as an indicator of the DM layer formation (Hu et al., 2016). Herein, the turbidity of both DMBRs decreased rapidly below 1 NTU within 5-20 min, while the DM layer formation time in PAC-DMBR was slightly shorter than that in C-DMBR. Subsequently, the turbidity in the stable filtration stage was almost the same $(<1$ NTU) in the two DMBRs. This finding indicated that PAC addition did not deteriorate the effluent turbidity but enhanced the DM layer formation in the PAC-DMBR.

In practical application, air backwashing was carried out when the flux declined to $10 \%$ of the initial flux. However, in this study, the air backwashing of the PAC-DMBR was carried out at flux levels slightly higher than that of $10 \%$ of the initial flux because of operational convenience and also for comparisons with the C-DMBR system. Thus, it was expected that the PACDMBR could further run for at least several hours or days additionally before the flux completely declined to $10 \%$ of the initial flux. From the operation results, it was considered that PAC addition could prolong the stable operational time of the DMBR system, which could reflect the improvement of the DM stability by PAC addition.

Furthermore, scanning electron microscope (SEM) measurement was carried out to analyze the morphology and structure of the DM layers. The SEM pictures of the DM layers are shown in Appendix A Fig. S3. It was found that a dense and evenly distributed DM layer with less porosity and little roughness was observed in the C-DMBR, while in the PAC-DMBR a less dense and uneven DM layer was formed on the mesh surface with 


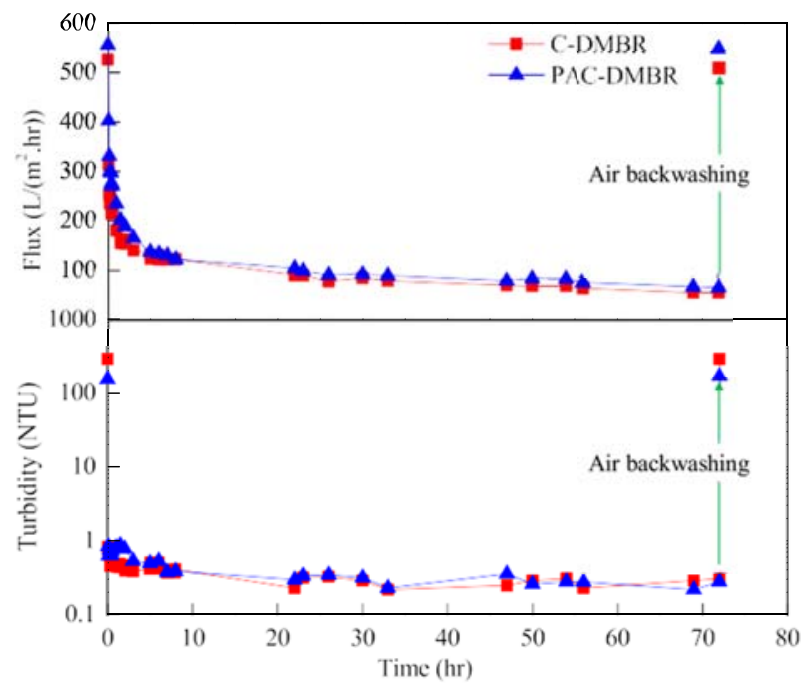

Fig. 1 - Variation profiles of flux and effluent turbidity during one operational cycle in the C-DMBR and PAC-DMBR.

high porosity and roughness. The reason for these differences was probably because PAC particles had higher mechanical robustness and incompressibility than sludge particles, which was beneficial for resisting the effects of hydraulic pressure and shear force on the DM layer in the PAC-DMBR. In this regard, it was believed that PAC addition could improve the DM stability.

\subsection{Morphological characteristics}

The morphology of the sludge flocs was characterized by PSD and shape. For the PSD, it was reported that PAC addition at $1 \mathrm{~g} / \mathrm{L}$ could increase the sludge particle size (Hu et al., 2017; $\mathrm{Ng}$ et al., 2006), which was confirmed by Fig. 2. More large

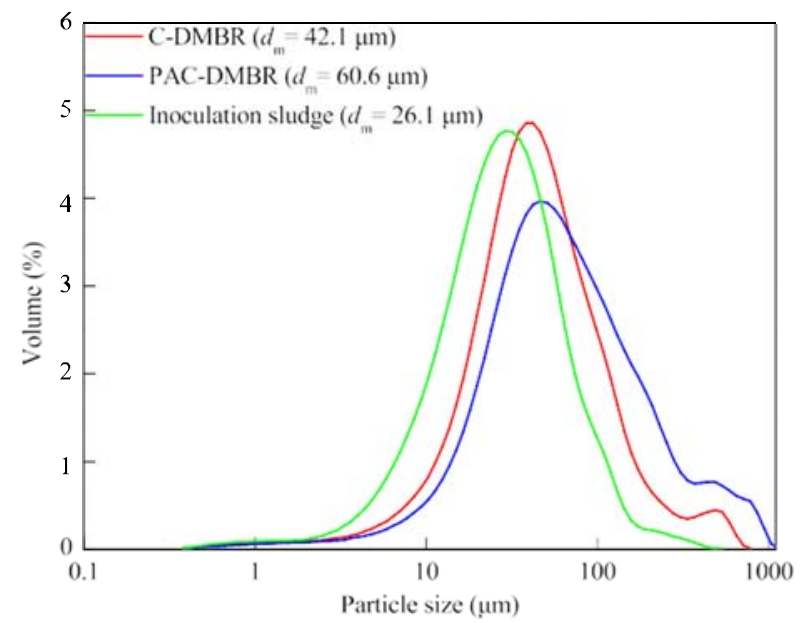

Fig. 2 - PSD of the inoculation sludge, PAC-DMBR sludge and C-DMBR sludge. Three replicate measurements were carried out for each sample with a standard deviation of less than 5\%, and typical profiles were plotted and reported. particles existing in the PAC-DMBR sludge was possibly because PAC particles could adsorb biopolymers, dissolved and colloidal organics and free bacterial cells from bulk solution, and also be readily integrated into the sludge flocs (forming BPAC). On the other hand, the incompressibility of PAC particles had a certain effect on the mechanical strength of sludge flocs against the hydraulic pressure and shear force, and thus, the sludge with PAC addition retained large size and strong structure. Furthermore, as reported, in the DM layer formation process, the flocs with size similar to or larger than the aperture of the support mesh were retained first by the support mesh to form the inner layer. Then, the smaller flocs were intercepted by this initial layer to form outer layers through a penetration-retention process in the DMBR, which was different from the deposition process of the cake layer in the conventional MBR (Chu et al., 2013). Therefore, the PACDMBR sludge with more large particles could more readily form a DM layer than the C-DMBR sludge, and which would develop the DM layer with higher porosity and lower compressibility to decrease filtration resistance and to improve flux in the PAC-DMBR system.

Typical microscopy observations of the sludge samples are shown in Appendix A Fig. S4. The fractal theory and image analysis were conducted based on the microscopy observations characterizing the sludge shape. Table 3 lists the shape parameters ( $D_{\mathrm{p}}, \mathrm{Ro}, \mathrm{FF}$, and $A R$ ) of sludge flocs in the two DMBRs. As shown in Table 3 , the $\mathrm{D}_{\mathrm{P}}$ of the PAC-DMBR sludge (1.17) was smaller than that of the C-DMBR sludge (1.32). This indicated that the sludge with PAC addition had a more regular shape than the sludge without PAC addition. This result also indicated that the sludge in the PAC-DMBR showed relatively strong structure. Furthermore, there was an increase in Ro for the PAC-DMBR sludge, implying that the shape of the sludge with PAC addition was relatively circular. Moreover, a decrease in AR and an increase in FF for the PACDMBR sludge also showed that the shape of sludge flocs became more regular due to PAC addition in the PAC-DMBR. Regular-shaped flocs were formed possibly because the biofilm with a relatively smooth contour was formed to surround the PAC particles due to the adsorption effect of the added PAC. As reported, sludge with irregular shape could develop a thick and dense cake layer on the membrane surface, which could significantly impede the membrane filtration process (Meng et al., 2006). Therefore, the modified morphology of sludge flocs by PAC addition was verified to exert some favorable influence on the structure and permeability of the DM layer.

Table 3 - Comparison of floc shape parameters between the C-DMBR sludge and PAC-DMBR sludge.

\begin{tabular}{lcccc} 
& \multicolumn{4}{c}{ Items } \\
\cline { 2 - 5 } & $\mathrm{D}_{\mathrm{P}}$ & $\mathrm{Ro}^{\mathrm{a}}$ & $\mathrm{FF}^{\mathrm{a}}$ & $\mathrm{AR}^{\mathrm{a}}$ \\
\hline C-DMBR & 1.32 & $0.34 \pm 0.09$ & $0.13 \pm 0.07$ & $2.23 \pm 0.77$ \\
PAC-DMBR & 1.17 & $0.49 \pm 0.13$ & $0.30 \pm 0.12$ & $1.96 \pm 0.57$ \\
\hline
\end{tabular}

a Ro, FF, and AR was given as average one \pm standard deviation; $\mathrm{n}=50$. 
Table 4 - Surface thermodynamic parameters and interaction energies per unit area between the sludge cells at contact

\begin{tabular}{|c|c|c|c|c|c|c|c|}
\hline \multirow[t]{2}{*}{ Sludge sample } & \multicolumn{4}{|c|}{ Surface thermodynamics parameters $\left(\mathrm{mJ} / \mathrm{m}^{2}\right)^{\mathrm{b}}$} & \multicolumn{3}{|c|}{ Interaction energy per unit area $\left(\mathrm{mJ} / \mathrm{m}^{2}\right)^{\mathrm{c}}$} \\
\hline & $\gamma^{\mathrm{LW}}$ & $\gamma^{+}$ & $\gamma^{-}$ & $\gamma^{\text {Tol }}$ & $\Delta G_{\mathrm{adh}}^{\mathrm{LW}}$ & $\Delta \mathrm{G}_{\mathrm{adh}}^{\mathrm{AB}}$ & $\Delta G_{\mathrm{adh}}$ \\
\hline C-DMBR & 29.65 & 0.32 & 24.83 & 35.27 & -1.20 & -1.20 & -2.40 \\
\hline PAC-DMBR & 33.13 & 0.25 & 19.92 & 37.56 & -2.36 & -10.68 & -13.04 \\
\hline
\end{tabular}

a The data was calculated through the average contact angle $(\theta)$ and average zeta potential ( $\xi$ ) by XDLVO theory. $\theta$ was the average value of at least eight measurements, while $\xi$ was the average value of at least six measurements.

${ }^{\mathrm{b}} \gamma^{\mathrm{LW}}, \gamma^{+}, \gamma^{-}$, and $\gamma^{\mathrm{Tol}}$ signified van der Waals, electron acceptor, electron donor components of the surface tension and total surface tension parameter, respectively.

c $\Delta G_{a d h}^{L W}, \Delta G_{a d h}^{A B}$, and $\Delta G_{\text {adh }}$ signified Lifshitz-van der Waals free energy, Lewis acid-based free energy and total interaction free energy between the sludge cells, respectively.

\subsection{Aggregation ability of sludge flocs}

Aggregation ability could be used as a comprehensive indicator of sludge properties because it was largely correlated to the EPS, structure, flocculability, settleability and dewaterability, adsorption ability, etc. of sludge flocs in the conventional activated sludge process and MBRs (Sheng et al., 2010). In this study, the sludge aggregation ability was assessed by XDLVO theory, which was calculated based on the contact angle and zeta potential as shown in Appendix A Table S2.

The involved surface thermodynamic parameters and interaction energies for aggregation ability were calculated by Appendix A Eqs. (S1)-(S7) and listed in Table 4. By comparison of surface thermodynamic parameters $\left(\mathrm{Y}^{\mathrm{LW}}, \mathrm{Y}^{+}, \mathrm{Y}^{-}\right.$and $\left.\mathrm{Y}^{\mathrm{Tol}}\right)$, it could be seen that PAC addition changed the sludge surface characteristics. On the other hand, the total interaction energy per unit area $\left(\Delta G_{\text {adh }}\right)$ of the PAC-DMBR sludge was more negative than that of the C-DMBR sludge, implying that the PAC-DMBR sludge showed a stronger attractive interaction when the sludge cells were adhering to each other. Additionally, $\Delta G_{\text {adh }}$, which represents the free energy of interaction between two identical surfaces immersed in water, could be used to estimate the surface hydrophobicity/hydrophilicity quantitatively (van Oss, 2003). If $\Delta \mathrm{G}_{\text {adh }}<0$, the surface is considered to be hydrophobic, otherwise $\left(\Delta G_{\text {adh }}>0\right)$, the surface is considered to be hydrophilic. According to this definition, the PAC-DMBR sludge with a highly negative value of $\Delta \mathrm{G}_{\text {adh }}$ was more hydrophobic than the C-DMBR sludge, indicating that PAC addition altered the sludge hydrophobicity.

In order to get more information, the profiles of total interaction energy with separation distance for the two sludge samples are shown in Fig. 3. The total interaction energy curves for both the PAC-DMBR sludge and C-DMBR sludge encountered an energy barrier and a secondary energy minimum. Generally, a higher energy barrier signifies the dispersed sludge cells in suspension should have a higher kinetic energy to overcome this barrier to reflocculate (Liu et al., 2010). The secondary energy minimum represents the ability of sludge cell to desorb from the sludge surface (Redman et al., 2004). In other words, a higher absolute value of the secondary energy minimum indicates that the sludge structure is more stable. From Fig. 3, the secondary energy minimum respectively was $-3.9 \mathrm{KT}$ and $-8.4 \mathrm{KT}$, while the energy barrier was $850.2 \mathrm{KT}$ and $505.2 \mathrm{KT}$ for the C-DMBR sludge and PAC-DMBR sludge, respectively. A reduction of the energy barrier and an increase of the secondary energy minimum (absolute value) were observed in the PAC-DMBR sludge.
These results indicated that PAC addition improved the sludge aggregation ability and structure, thereby protecting large sludge flocs from being broken under external forces (e.g., shear force) (Remy et al., 2010). Similarly, the smaller size (1-10 $\mu \mathrm{m})$ of sludge flocs resulting from the decreased aggregation was also reported in a previous study (Wang et al., 2011). The above findings indicated that improved aggregation ability was possibly beneficial for the larger and more stable sludge flocs in the PAC-DMBR, which further confirmed the description of the sludge morphology as discussed in Section 2.2. Furthermore, the sludge with stable structure potentially reduced the release of EPS from the sludge flocs to the bulk solution, which could alleviate the blocking of the DM layer and physical irremovable fouling of the DMBRs (Hu et al., 2017). Also, Su et al. (2013) suggested that sludge with poor aggregation ability had higher mem- brane fouling potential. Consequently, the sludge with good aggregation ability in the PAC-DMBR showed a lower DM layer fouling potential and higher DM layer permeability.

\subsection{Microbial community characterization}

\subsubsection{Microbial metabolic analysis}

Biolog assay was applied to evaluate the metabolic characteristics of the microbial communities in the activated sludge. As shown in Fig. 4a, the evolutions of the AWCD in the PAC-DMBR sludge

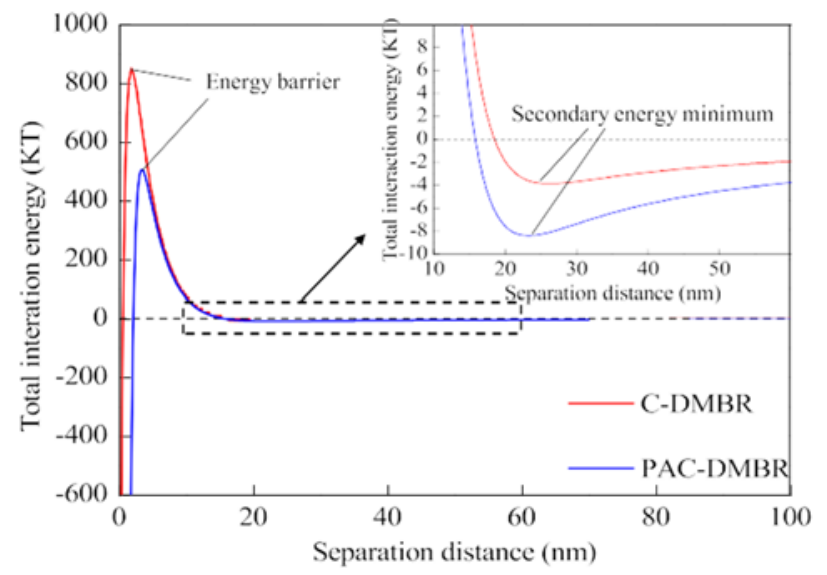

Fig. 3 - Total interaction energy curves as a function of separation distance for the C-DMBR sludge and PAC-DMBR sludge. 

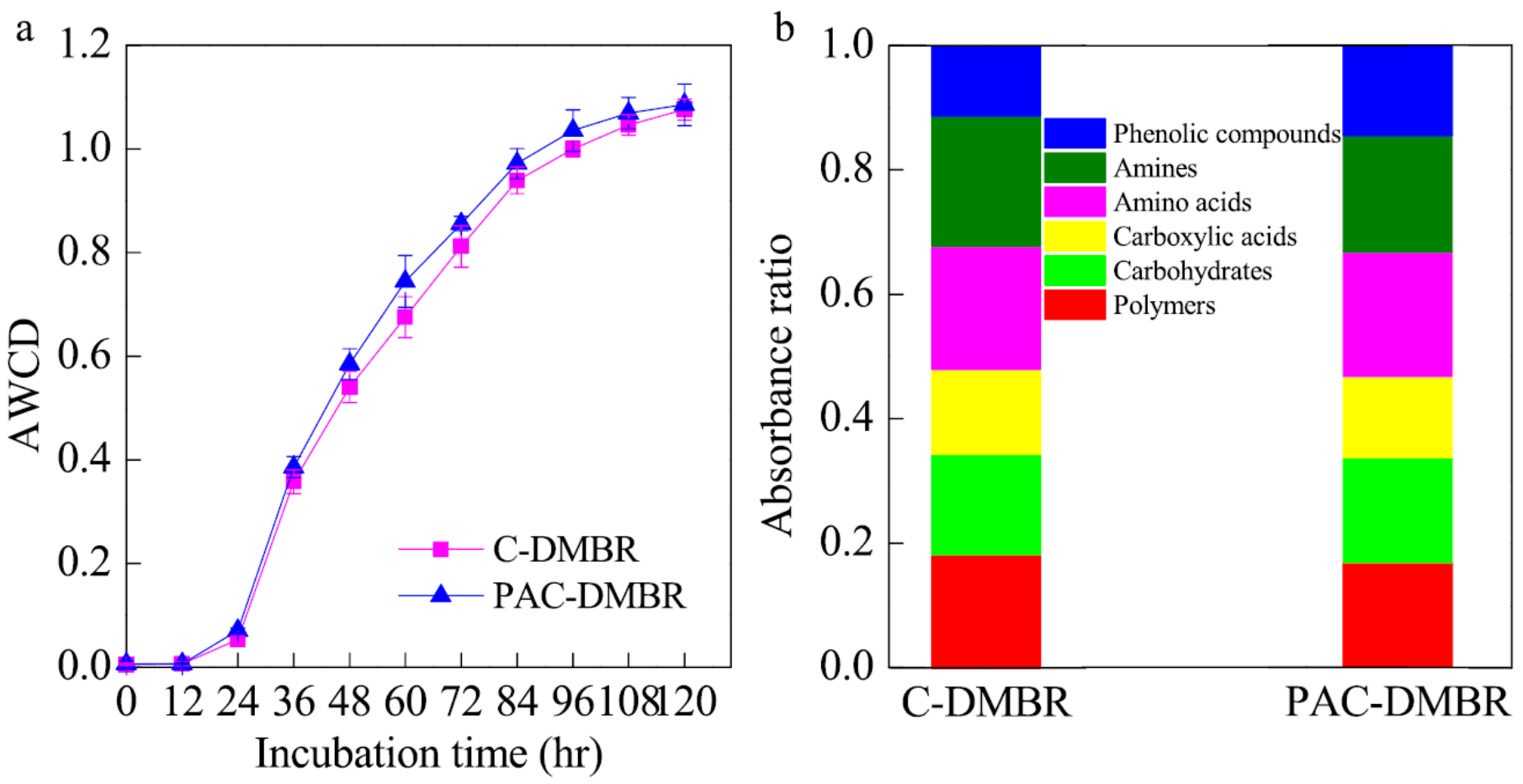

Fig. 4 - Aaverage well color development (AWCD) (a) and average absorbance ratio (b) of carbon source in Biolog EcoPlate ${ }^{\mathrm{TM}}$ for the C-DMBR sludge and PAC-DMBR sludge. The error bars signified the standard deviation of three replicate measurements.

and C-DMBR sludge were insignificant in the initial 0-12 hr. Subsequently, the AWCD rapidly increased after this lag phase, and finally approached the value of 1.1 at the end-point of the incubation period. Furthermore, it was found that the AWCD in the PAC-DMBR sludge was slightly higher than that in the CDMBR sludge during the $12-120 \mathrm{hr}$ (Fig. 4a). This difference indicated that PAC addition at $1 \mathrm{~g} / \mathrm{L}$ dosage could slightly enhance the microbial metabolic activity in the DMBR. It might be due to that BPAC particles facilitated the adsorption of microbial cells, enzymes and organic matters, which, in turn, provided a suitable environment for microbial metabolism (Zhang et al., 1991). On the other hand, the higher microbial activity might be from the higher diversity of microbial communities, which would be further discussed in the "microbial community analysis" section.

In addition, the average absorbance ratio $\left(\mathrm{F}_{\mathrm{j}}\right)$ of each carbon category at $120 \mathrm{hr}$ in the two sludge samples is presented in Fig. 4b. From Fig. 4b, the microbial communities in the two sludge samples showed higher metabolic activity for amines, amino acids, carbohydrates and polymers $\left(F_{j}>0.15\right)$ as compared with carboxylic acids and phenolic compounds $\left(F_{j}<0.15\right)$. Overall, for the two sludge samples, there was no significant difference in the metabolic properties of microbial communities on the different carbon category. This finding indicated that PAC addition had no significant influence on the microbial community metabolic structures.

\subsubsection{Microbial community analysis}

As mentioned, the presences of eukaryotes (such as protists and metazoans) played an important role in the sludge properties and systems performance. Generally, in activated sludge the common protists include flagellates, amoebae, ciliates and heliozoans, while the metazoans include rotifers, nematoeds and oligochaetes. Direct microscopic observations of the two sludge samples are shown in Appendix A Fig. S4. Ciliates, amoebae and rotifers were observed in the two sludge samples. The numbers of these eukaryotes ranged from some dozens to hundreds per mL. Relatively, rotifers were less abundant than the other two microorganisms (ciliates and amoebae). Only a small amounts of rotifers in the two sludge samples indicated that the activated sludges were in the normal physiological state. Moreover, above analysis indicated that PAC addition would not exert very significant influence on the propagation of eukaryotes (pro- tists and metazoans) in the PAC-DMBR. Additionally, higher invertebrates such as nematoeds and oligochaetes, which

Table 5 - Richness and diversity of microbial communities in the C-DMBR sludge and PAC-DMBR sludge.

\begin{tabular}{lllllll} 
Sample & Sequences & OTUs & Chao1 $^{\mathrm{a}}$ & Shannon $^{\mathrm{b}}$ & Simpson $^{\mathrm{c}}$ & Coverage $^{\mathrm{d}}$ \\
\hline C-DMBR & 25,121 & 1358 & 1822 & 4.096 & 0.136 & 0.980 \\
PAC-DMBR & 32,325 & 1735 & 2300 & 4.976 & 0.045 & 0.981 \\
\hline
\end{tabular}

DMBR: dynamic membrane bioreactor; PAC: powdered activated carbon; OTUs: operational taxonomic units.

a A higher number of Chao 1 indicated higher microbial richness.

b A higher number of Shannon signified more microbial diversity.

c A lower number of Simpson represented more microbial diversity.

${ }^{\mathrm{d}}$ Coverage estimated probability that the next read will belong to an OTU that has already been found. 
acted as the predators to lead to the improvement of permeate flux (Klein et al., 2016), did not observe in the two DMBRs. So, much more attentions should be paid to this aspect in the long-term operation of PAC-DMBR system.

On the other hand, the sludge samples were collected from the two DMBRs at the end of the operation and their DNA was extracted for high-throughput pyrosequencing. Based on the sequencing results of bacterial 16S rRNA gene in Table 5, a total of 1358 (C-DMBR) and 1735 (PAC-DMBR) operational taxonomic units (OTUs) were clustered at a 97\% sequence similarity, which indicated that the bacterial diversity was increased in the PACDMBR sludge. A further comparison of the Chao 1 index, Shannon index and Simpson index showed greater bacterial richness and diversity in the PAC-DMBR than that in the C-
DMBR. This phenomenon was probably attributed to PAC addition creating additional microenvironment in the form of BPAC for the enrichment and growth of some specific bacteria.

Furthermore, the bacterial communities at the phylum level for the two sludge samples are shown in Appendix A Fig. S5. The microbial communities of total bacteria were grouped into 19 identified phyla, among which Proteobacteria was the predominant phylum, accounting for $74.7 \%$ and $67.0 \%$ in the C-DMBR sludge and PAC-DMBR sludge, respectively. Phyla Bacteroidetes, Planctomycetes, Firmicutes and Choroflexi were the subdominant groups, which were respectively at $4.6 \%, 11.4 \%$, $1.6 \%, 2.1 \%$ in the C-DMBR sludge and $9.2 \%, 6.4 \%, 10.0 \%, 2.9 \%$ in the PAC-DMBR sludge. The above five bacterial phyla were ubiquitous in biological wastewater treatment reactors, which

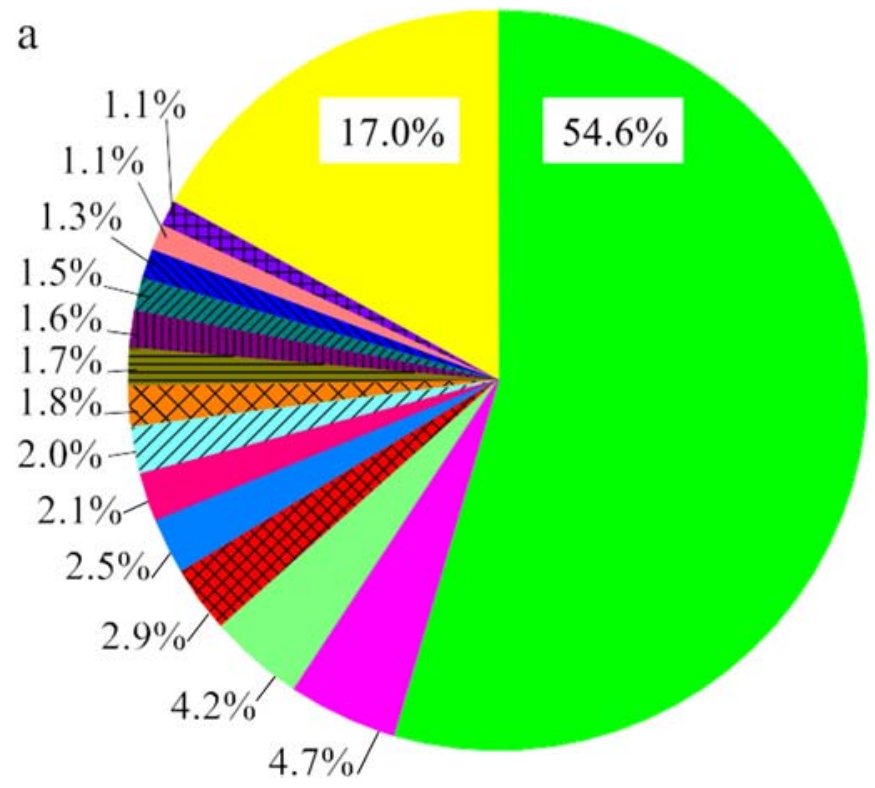

Unclassified
Thiobacillus
Simplicispira
BDI-7_Clade
CL500-3
Nitrobacter
VII__Arenimonas
Xlanctomyces
Acidovorax
Candidatus_Alysiosphaera
Reyranella
SM1A02
Thauera
Sva0081_sediment_group
Others

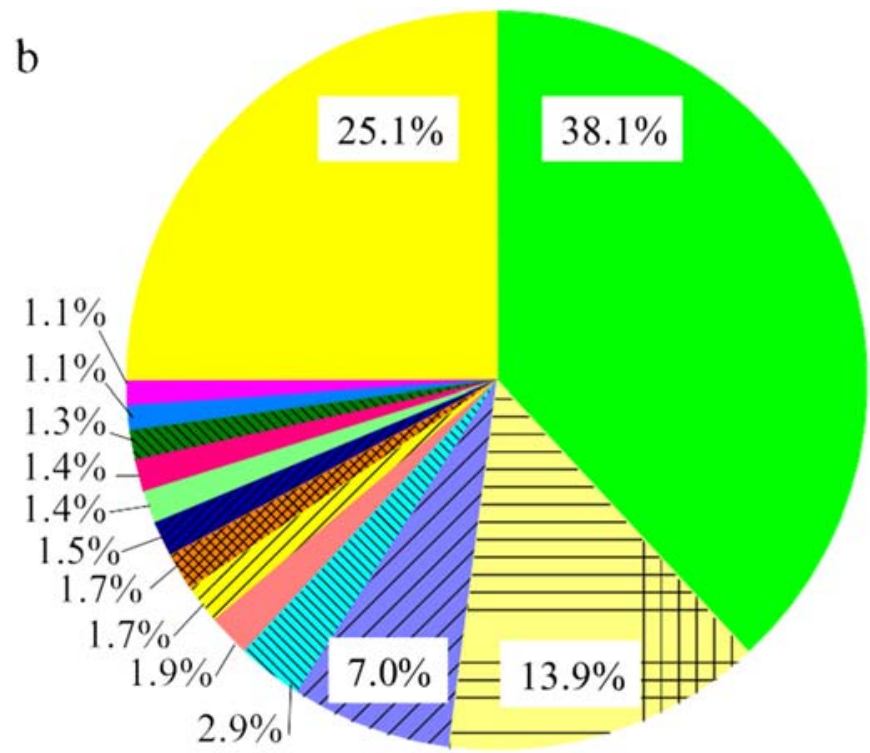

Unclassified
A.IInetobacter
Thauera
Cloacibacterium
Clostridium_sensu_stricto_13
Simplicispira
Nitrobacter
Nitrospira
CL500-3
Thiobacillus
Others

Fig. 5 - Composition and abundance of bacterial communities at genus level in the C-DMBR sludge (a) and PAC-DMBR sludge (b). 
could degrade and transform organics (Ma et al., 2013a, 2013b). Overall, the composition of bacterial phyla in the two sludge samples was nearly identical, while the relative abundance of phyla, especially some dominant phyla, was notably altered due to PAC addition.

To further elucidate the functions of bacterial communities in the sludge samples, the further classification at the genus level is shown in Fig. 5. It was noteworthy that the genus with a relative abundance of less than $1.0 \%$ was assigned to "Others", and the total of genera in "Other" were 176 and 230 for C-DMBR sludge and PAC-DMBR sludge, respectively. By comparison, it was found that the dominant genera in the PAC-DMBR sludge were quite different from those in the C-DMBR sludge, indicating that there was an evolution of the bacterial community after PAC addition in the DMBR system. In detail, genera Acinetobacter (13.9\%), Aeromonas (7.0\%), and Comamonas (2.9\%) were highly enriched in the PAC-DMBR sludge rather than in the C-DMBR sludge. Genus Aeromonas belonging to the facultative anaerobic bacteria was enriched in the PAC-DMBR indicating that a local facultative anaerobic/anaerobic microenvironment was probably created in the sludge with PAC addition. Genus Acinetobacter belonging to the wider class of Gammaproteobacteria could adhere onto both biological and abiotic surface, and some members of Acinetobacter could function as bridging organisms in floc formation in the activated sludge process (Doughari et al., 2011; Phuong et al., 2012). Similarly, some members of Comamonas were reported as the floc-forming bacteria in activated sludge (Tago and Yokota, 2004). Genera Flavobacterium and Pseudomonas were also considered as the common floc-forming bacteria, which were able to synthesize extracellular materials to bind microbial cells, colloids, organic polymers, cations and inorganics together to form activated sludge flocs (Gerardi, 2006; Kato et al., 1971). However, the relative abundance of Flavobacterium and Pseudomonas (belonging to genus "Others") was very low, which were $0.31 \%$ and $0.62 \%$ in the PAC-DMBR sludge, and $0.09 \%$ and $0.41 \%$ in the CDMBR sludge. The above-mentioned bacteria contributing to sludge floc formation would further affect the sludge morphology (such as floc size, shape and structure) and aggregation ability.
Also, their enrichments in the sludge with PAC addition implied that PAC particles could provide a fixed surface for microbial growth as the bio-carrier. Moreover, the highly enriched Acinetobacter (13.9\%) also has the capability of degrading hydrocarbons (especially for the aromatic compounds) (Margesin et al., 2003), which could have potentially contributed to the removal of the relatively complex organics in the PAC-DMBR system.

2.5. Effects of sludge properties on the performance enhancement by PAC addition

As reported, the sludge properties were considered to be an important factor to affect the operation of the DMBR process (Liang et al., 2013). In this study, the PAC addition significantly modified the sludge morphology (Fig. 2 and Table 3), aggregation ability (Fig. 3), and microbial communities (particularly in bacterial communities) (Fig. 5 and Table 5) in the PAC-DMBR. Due to little differences were observed in the presence of eukaryotes (protists and metazoans) for the two DMBRs, the analysis of the eukaryotic community composition was not performed.

Synthetically, the effects of PAC addition on the sludge properties and subsequent enhancement of the performance in the PAC-DMBR are further discussed and schematically shown in Fig. 6. The adsorption and incompressibility of PAC particles played an important role in altering the sludge morphology so that sludge flocs with large size, regular shape and strengthened structure were greatly formed in the PAC-DMBR system. Moreover, PAC addition modified the surface thermodynamic parameters (such as $\mathrm{Y}^{+}, \mathrm{Y}^{-}, \mathrm{Y}^{\mathrm{LW}}$ and $\mathrm{Y}^{\mathrm{Tol}}$ ) and hydrophobicity of sludge flocs due to the hydrophobicity of PAC particle, which contributed to improving the sludge aggregation ability (Hou et al., 2015). Consequently, the sludge with good aggregation ability strengthened the sludge structure, increased the sludge size, and induced the reduction of biopolymers (such as EPS) release. Finally, PAC addition to the DMBR could create the additional microenvironment and serve as the bio-carrier for microbial growth, hence enriching some bacteria (such as genera

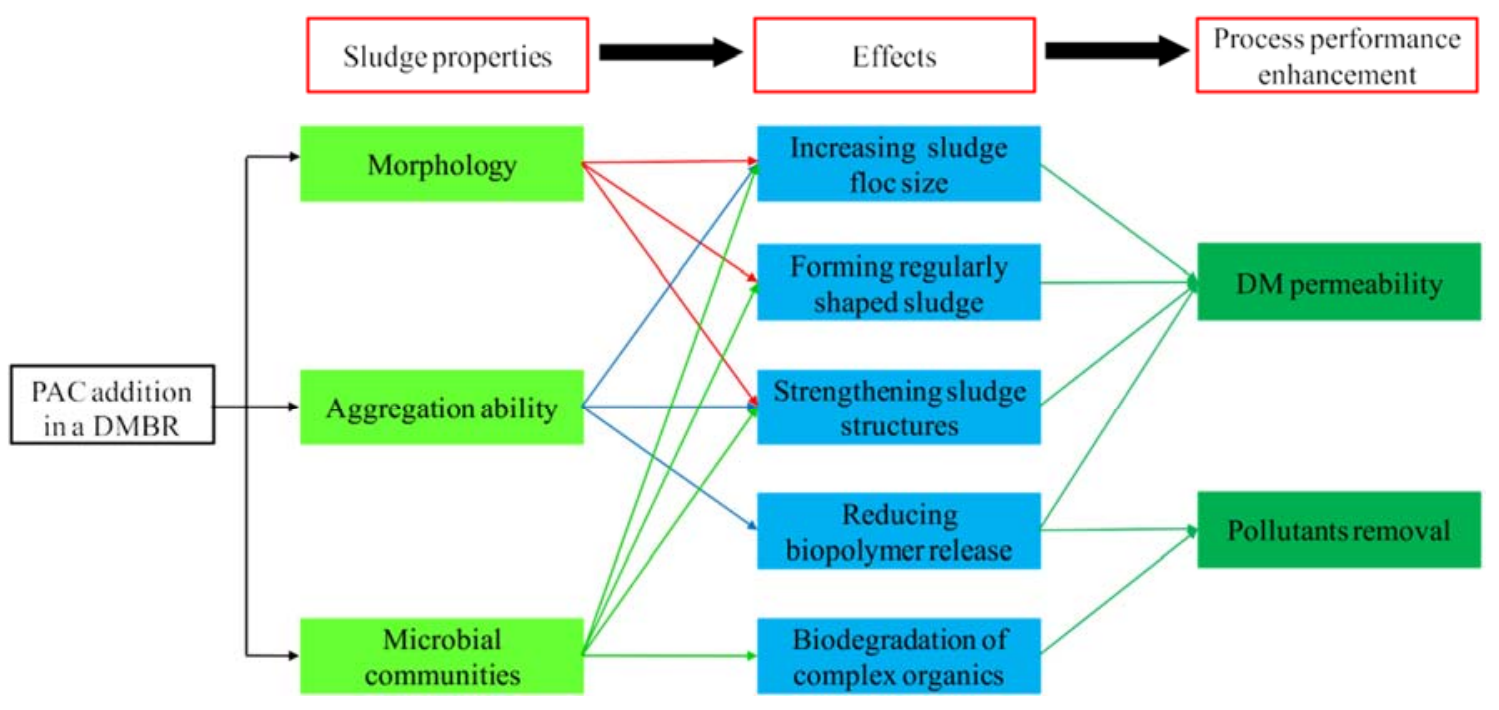

Fig. 6 - Schematic illustration of performance enhancement by PAC addition in the DMBR. 
Acinetobacter, Comamonas, Flavobacterium and Pseudomonas) with specific floc formation functions. Among various bacterial genera, the high enrichment of Acinetobacter was responsible for the biodegradation of complex organics. Additionally, Fig. 6 also reveals that complex interactions existed among the three sludge properties, e.g., sludge morphology, aggregation ability and microbial communities. As discussed previously, PAC addition in the DMBR modified the sludge properties through the following five specific aspects: increasing sludge size, forming regularly shaped sludge, strengthening sludge struc- tures, reducing biopolymer release and biodegradation of complex organics.

The modifications of the aforementioned first four sludge properties were expected to further improve the incompressibility and porosity of DM layer, which was evidenced by the discussion in Section 2.1 regarding the morphology and structure of the DM layer. From this result it followed that the less dense and more porous of DM layer in the PACDMBR was contributed to the improvement of DM permeability. Simultaneously, DM layer as the "secondary" membrane could reject a small fraction of the pollutants. Moreover, PAC particles could adsorb organics from bulk solution, and also be readily integrated into the sludge flocs to form BPAC. These adsorbed organics could be biodegraded by the attached microbial communities on the BPAC surface (Hu et al., 2017; Skouteris et al., 2015). Additionally, good sludge aggregation ability in the PAC-DMBR potentially reduced the release of organics (in particularly biopolymer), which was also beneficial for the pollutants removal. Overall, PAC addition modified sludge properties and contributed to enhancing the process performance of the PAC-DMBR in terms of DM permeability and pollutants removal.

\section{Conclusion}

In this study, the effects of PAC addition on the sludge properties in the PAC-DMBR hybrid process were investigated while the mechanisms of performance enhancement were explored. The results demonstrated that the PAC-DMBR system was an effective and feasible wastewater treatment to achieve enhanced pollutants removal and improved filtration performance. Moreover, the adsorption effect, incompressibility and hydrophobicity nature, and bio-carrier property of PAC played an important role in the modifications of sludge properties (such as morphology, aggregation ability and microbial communities). These modifications were evidenced by the formation of larger, more regularly shaped and strengthened sludge flocs, the improvement of sludge aggregation ability and bacterial diversity, which further affected the morphology, structure and permeability of the DM layer and thus enhanced the DMBR performance.

\section{Acknowledgments}

This work was supported by the National Natural Science Foundation of China (Nos. 51778522, and 51508450), and the Program for Innovative Research Team in Shaanxi (No. IRT2013KCT-13).
Appendix A. Supplementary data

Supplementary data to this article can be found online at https://doi.org/10.1016/j.jes.2018.03.003.

\section{R E F E R E N C E S}

Achmadulina, F.Y., Zakirov, R.K., Balymova, E.S., Denisova, V., Brovdyová, T., Trögl, J., et al., 2017. Comparison of bioindicator eukaryotes of activated sludge biocenoses on two water-treatment plants: a case study. Nova. Biotechnol. Chim. 16 (1), 54-60

Cai, X., Zhang, M., Yang, L., Lin, H., Wu, X., He, Y., et al., 2017. Quantification of interfacial interactions between a rough sludge floc and membrane surface in a membrane bioreactor. J. Colloid Interface Sci. 490, 710-718,

Cenens, C., van Beurden, K.P., Jenné, R., Van Impe, J.F., 2002. On the development of a novel image analysis technique to distinguish between flocs and filaments in activated sludge images. Water Sci. Technol.46(1-2), 381-387.

Chinese, N.E.P.A, 2002. Water and Wastewater Monitoring Methods. Chinese Environmental Science Publishing House, Beijing, China.

Choi, K.H., Dobbs, F.C., 1999. Comparison of two kinds of Biolog microplates (GN and ECO) in their ability to distinguish among aquatic microbial communities. J. Microbiol. Methods 36 (3), 203-213.

Chu, H., Zhang, Y., Zhou, X., Dong, B., 2013. Bio-enhanced powder-activated carbon dynamic membrane reactor for municipal wastewater treatment. J. Membr. Sci. 433, 126-134.

Derlon, N., Koch, N., Eugster, B., Posch, T., Pernthaler, J., Pronk, W., et al., 2013. Activity of metazoa governs biofilm structure formation and enhances permeate flux during Gravity-Driven Membrane (GDM) filtration. Water Res. 47 (6), 2085-2095.

Doughari, H.J., Ndakidemi, P.A., Human, I.S., Benade, S., 2011. The ecology, biology and pathogenesis of Acinetobacter spp.: an overview. Microbes Environ. 26 (2), 101-112.

Elfstrand, S., Hedlund, K., Mårtensson, A., 2007. Soil enzyme activities, microbial community composition and function after 47 years of continuous green manuring. Appl. Soil Ecol. 35 (3), 610-621.

Ersahin, M.E., Ozgun, H., Dereli, R.K., Ozturk, I., Roest, K., van Lier, J.B., 2012. A review on dynamic membrane filtration: materials, applications and future perspectives. Bioresour. Technol. 122 (5), 196-206

Gerardi, M.H., 2006. Floc-forming bacteria. Wastewater Bacteria. John Wiley \& Sons, Inc., Hoboken, NJ, USA, pp. 133-141.

Hong, H., Lin, H., Mei, R., Zhou, X., Liao, B.Q., Zhao, L., 2016. Membrane fouling in a membrane bioreactor: a novel method for membrane surface morphology construction and its application in interaction energy assessment. J. Membr. Sci. 516, 135-143.

Hong, H., Cai, X., Shen, L., Li, R., Lin, H., 2017. Membrane fouling in a submerged membrane bioreactor: new method and its applications in interfacial interaction quantification. Bioresour. Technol. 241, 406-414.

Hou, X., Liu, S., Zhang, Z., 2015. Role of extracellular polymeric substance in determining the high aggregation ability of anammox sludge. Water Res. 75, 51-62.

Hu, Y., Wang, X.C., Tian, W., Ngo, H.H., Chen, R., 2016. Towards stable operation of a dynamic membrane bioreactor (DMBR): operational process, behavior and retention effect of dynamic membrane. J. Membr. Sci. 498, 20-29.

Hu, Y., Wang, X.C., Sun, Q., Ngo, H.H., Yu, Z., Tang, J., et al., 2017. Characterization of a hybrid powdered activated 
carbon-dynamic membrane bioreactor (PAC-DMBR) process with high flux by gravity flow: operational performance and sludge properties. Bioresour. Technol. 223, 65-73.

Jenkins, D., Richard, M.G., Daigger, G.T., 2004. Manual on the Causes and Control of Activated Sludge Bulking, Foaming, and Other Solids Separation Problems. third ed. IWA Publishing, London, UK.

Kato, A., Izaki, K., Takahashi, H., 1971. Floc-forming bacteria isolated from activated sludge. J. Gen. Appl. Microbiol. 17 (6), 439-456.

Kiso, Y., Jung, Y.J., Ichinari, T., Park, M., Kitao, T., Nishimura, K., et al., 2000. Wastewater treatment performance of a filtration bio-reactor equipped with a mesh as a filter material. Water Res. 34 (17), 4143-4150.

Klein, T., Zihlmann, D., Derlon, N., Isaacson, C., Szivak, I., Weissbrodt, D.G., et al., 2016. Biological control of biofilms on membranes by metazoans. Water Res. 88, 20-29.

Liang, S., Qu, L., Meng, F., Han, X., Zhang, J., 2013. Effect of sludge properties on the filtration characteristics of self-forming dynamic membranes (SFDMs) in aerobic bioreactors: formation time, filtration resistance, and fouling propensity. J. Membr. Sci. 436 (2), 186-194.

Lin, C.K., Tsai, T.Y., Liu, J.C., Chen, M.C., 2001. Enhanced biodegradation of petrochemical wastewater using ozonation and BAC advanced treatment system. Water Res. 35 (3), 699704.

Liu, X.M., Sheng, G.P., Luo, H.W., Zhang, F., Yuan, S.J., Xu, J., et al., 2010. Contribution of extracellular polymeric substances (EPS) to the sludge aggregation. Environ. Sci. Technol. 44 (11), 43554360.

Ma, J., Wang, Z., Yang, Y., Mei, X., Wu, Z., 2013a. Correlating microbial community structure and composition with aeration intensity in submerged membrane bioreactors by 454 high-throughput pyrosequencing. Water Res. 47 (2), 859-869.

Ma, J., Wang, Z., Zou, X., Feng, J., Wu, Z., 2013b. Microbial communities in an anaerobic dynamic membrane bioreactor (AnDMBR) for municipal wastewater treatment: Comparison of bulk sludge and cake layer. Process Biochem. 48 (3), 510-516.

Margesin, R., Labbe, D., Schinner, F., Greer, C.W., Whyte, L.G., 2003. Characterization of hydrocarbon-degrading microbial populations in contaminated and pristine alpine soils. Appl. Environ. Microbiol. 69(6), 3085-3092.

Meng, F., Yang, F., Xiao, J., Zhang, H., Gong, Z., 2006. A new insight into membrane fouling mechanism during membrane filtration of bulking and normal sludge suspension. J. Membr. Sci. 285 (1-2), 159-165.

Ng, C.A., Sun, D., Fane, A.G., 2006. Operation of membrane bioreactor with powdered activated carbon addition. Sep. Purif. Technol. 41 (7), 1447-1466.

Phuong, K., Hanazaki, S., Kakii, K., Nikata, T., 2012. Involvement of Acinetobacter sp. in the floc-formation in activated sludge process. J. Biotechnol. 157 (4), 505-511.

Quek, P.J., Yeap, T.S., Ng, H.Y., 2017. Applicability of upflow anaerobic sludge blanket and dynamic membrane-coupled process for the treatment of municipal wastewater. Appl. Microbiol. Biotechnol. 101 (16), 6531-6540.

Redman, J.A., Walker, S.L., Elimelech, M., 2004. Bacterial adhesion and transport in porous media: role of the secondary energy minimum. Environ. Sci. Technol. 38 (6), 1777-1785.

Remy, M., Potier, V., Temmink, H., Rulkens, W., 2010. Why low powdered activated carbon addition reduces membrane fouling in MBRs. Water Res. 44 (3), 861-867.

Satyawali, Y., Balakrishnan, M., 2009. Effect of PAC addition on sludge properties in an MBR treating high strength wastewater. Water Res. 43 (6), 1577-1588.

Sheng, G.P., Yu, H.Q., Li, X.Y., 2010. Extracellular polymeric substances (EPS) of microbial aggregates in biological wastewater treatment systems: a review. Biotechnol. Adv. 28 (6), 882-894.
Skouteris, G., Saroj, D., Melidis, P., Hai, F.I., Ouki, S., 2015. The effect of activated carbon addition on membrane bioreactor processes for wastewater treatment and reclamation - a critical review. Bioresour. Technol. 185, 399-410.

Su, X., Tian, Y., Li, H., Wang, C., 2013. New insights into membrane fouling based on characterization of cake sludge and bulk sludge: an especial attention to sludge aggregation. Bioresour. Technol. 128 (1), 586-592.

Tago, Y., Yokota, A., 2004. Comamonas badia sp. nov., a floc-forming bacterium isolated from activated sludge. J. Gen. Appl. Microbiol. 50 (5), 243-248.

Tian, Y., Chen, L., Zhang, S., Zhang, S., 2011. A systematic study of soluble microbial products and their fouling impacts in membrane bioreactors. Chem. Eng. J. 168 (3), 1093-1102.

van Oss, C.J., 2003. Long-range and short-range mechanisms of hydrophobic attraction and hydrophilic repulsion in specific and aspecific interactions. J. Mol. Recognit. 16 (4), 177-190.

Wang, Q., Wang, Z., Wu, Z., Han, X., 2011. Sludge reduction and process performance in a submerged membrane bioreactor with aquatic worms. Chem. Eng. J. 172 (2-3), 929-935.

Whang, G.D., Cho, Y.M., Park, H., Jang, J.G., 2004. The removal of residual organic matter from biologically treated swine wastewater using membrane bioreactor process with powdered activated carbon. Water Sci. Technol. 49 (5-6), 451-457.

Wu, B., Christen, T., Tan, H.S., Hochstrasser, F., Suwarno, S.R., Liu, X., et al., 2017. Improved performance of gravity-driven membrane filtration for seawater pretreatment: implications of membrane module configuration. Water Res. 114, 59-68.

Yang, C., Zhang, W., Liu, R., Li, Q., Li, B., Wang, S., et al., 2011. Phylogenetic diversity and metabolic potential of activated sludge microbial communities in full-scale wastewater treatment plants. Environ. Sci. Technol. 45 (17), 7408-7415.

Yu, Y., Yang, Z., Duan, Y., 2017. Structure and flow calculation of cake layer on microfiltration membranes. J. Environ. Sci. 56 (6), 95-101.

Yuan, S., Gao, M., Ma, H., Afzal, M.Z., Wang, Y.K., Wang, M., et al., 2017. Qualitatively and quantitatively assessing the aggregation ability of sludge during aerobic granulation process combined XDLVO theory with physicochemical properties. J. Environ. Sci. https://doi.org/10.1016/j. jes.2017.08.015.

Zhang, X., Wang, Z., Gu, X., 1991. Simple combination of biodegradation and carbon adsorption - the mechanism of the biological activated carbon process. Water Res. 25 (2), 165-172.

Zhang, T.Y., Wu, Y.H., Zhuang, L.L., Wang, X.X., Hu, H.Y., 2014. Screening heterotrophic microalgal strains by using the Biolog method for biofuel production from organic wastewater. Algal Res. 6, 175-179.

Zhao, Y., Wu, K., Wang, Z., Zhao, L., Li, S., 2000. Fouling and cleaning of membrane - a literature review. J. Environ. Sci. 12 (2), 241-251.

Zhao, L., Yang, L., Lin, H., Zhang, M., Yu, H., Liao, B.Q., et al., 2016 a. Modeling three-dimensional surface morphology of biocake layer in a membrane bioreactor based on fractal geometry. Bioresour. Technol. 222, 478-484.

Zhao, L., Zhang, M., He, Y., Chen, J., Hong, H., Liao, B.Q., et al., 2016b. A new method for modeling rough membrane surface and calculation of interfacial interactions. Bioresour. Technol. 200 (2), 451-457.

Zhao, L., Qu, X., Lin, H., Yu, G., Liao, B.Q., 2018. Simulation of foulant bioparticle topography based on gaussian process and its implications for interface behavior research. Appl. Surf. Sci. 434, 975-981. 